\title{
Cervical Cancer Screening with AMIGAS:
}

\section{A Cost-Effectiveness Analysis}

\author{
David R. Lairson, PhD, Yu-Chia Chang, MPH, Theresa L. Byrd, DrPH, Judith Lee Smith, \\ PhD, Maria E. Fernandez, PhD, and Katherine M. Wilson, PhD \\ School of Public Health (Lairson, Chang, Fernandez), Center for Health Promotion and \\ Prevention Research (Fernandez), University of Texas Health Science Center at Houston, \\ Houston, Texas; Paul L. Foster School of Medicine (Byrd), Texas Tech University, Lubbock, \\ Texas; and the CDC, Division of Cancer Prevention and Control (Smith), Epidemiology and \\ Analysis Program Office (Wilson), Atlanta, Georgia
}

\section{Abstract \\ Background-Hispanic women have a higher incidence of cervical cancer than all other races and ethnicities. In Hispanic subgroups, Mexican American women were among the least likely to have received cervical cancer screening. In a recent RCT, Ayudando a las Mujeres con Información, Guia, y Amor para su Salud (AMIGAS) was shown to increase cervical cancer screening rates among women of Mexican descent at 6 months in all intervention arms compared to the control arm. Limited information exists about the economics of interventions to increase cervical cancer screening rates among women of Mexican descent.}

Purpose-This study aims to estimate the cost-effectiveness of the alternative AMIGAS intervention methods for increasing cervical cancer screening among low-income women of Mexican descent in three U.S. communities.

Methods-Cost data were collected from 2008 to 2011 alongside the AMIGAS study of 613 women. Receipt of Pap test within 6 months of intervention was the primary outcome measure in the cost-effectiveness analysis, conducted during 2012-2013.

Results-The cost per additional woman screened comparing the video-only intervention to usual care was $\$ 980$. The cost increased to $\$ 1,309$ with participant time cost included. With an additional cost per participant of $\$ 3.90$ compared to flipchart only, the full AMIGAS program (video plus flipchart) yielded $6.8 \%$ additional women screened.

Conclusions-Results on the average and incremental cost-effectiveness of the AMIGAS program elements may assist health policymakers and program managers to select and appropriately budget for interventions shown to increase cervical cancer screening among lowincome women of Mexican descent.

Address correspondence to: David R. Lairson, PhD, University of Texas Health Science Center at Houston School of Public Health, 1200 Herman Pressler, Houston TX 77030. david.r.lairson@uth.tmc.edu.

No financial disclosures were reported by the authors of this paper. 


\section{Introduction}

Women without a routine source of health care, ${ }^{1}$ of low education, ${ }^{2}$ and low income levels ${ }^{3}$ have higher rates of cervical cancer mortality. For average-risk women, the U.S. Preventive Services Task Force recommends regular Pap tests beginning at age 21 years and screening at least every 3 years (when using the Pap test alone). 4,5

In 2009, more than 12,000 women in the U.S. were diagnosed with cervical cancer, with Hispanic women having a higher incidence of cervical cancer than all other races and ethnicities. ${ }^{6}$ In Hispanic subgroups, Mexican American women were among the least likely to have received cervical cancer screening. ${ }^{7}$ Hispanic women and women living along the U.S.-Mexico border were more likely to die from this disease than others. ${ }^{8}$

Using effective interventions to address this inequality is a priority. ${ }^{9}$ The Healthy People 2020 objective for cervical cancer screening is to increase the percentage of women aged 21-65 years who have been screened to 93\%. ${ }^{9}$ The Affordable Care Act requires Medicare and most private insurance plans to cover certain recommended preventive services, including cervical cancer screening, with no cost sharing. The law will increase access to health insurance for millions of Americans who have not had a usual source of care and regular access to preventive services. ${ }^{10,11}$

Implementing effective interventions to educate and increase use of preventive services is necessary to achieve local and national cancer control and prevention goals. ${ }^{8,9}$ The Community Preventive Services Task Force found strong evidence to support the effectiveness of one-on-one education and small media strategies ${ }^{12}$ for increasing cervical cancer screening, ${ }^{13}$ and recommends these approaches. ${ }^{14}$

A recent report described the development ${ }^{15}$ and effectiveness of the Ayudando a las Mujeres con Información, Guia, y Amor para su Salud (AMIGAS) intervention in increasing Pap test screening among Mexican American women living in the U.S. ${ }^{16}$ This paper reports results of the cost-effectiveness analysis conducted as part of the AMI-GAS study to assess the incremental gain in cervical cancer screening compliance compared to the incremental cost of moving from a usual care control group to successively more involved interventions (i.e., video or flipchart, or video and flipchart delivered by a trained health worker).

Studies have provided evidence on the cost-effectiveness of screening and treatment for cervical cancer by screening interval and risk group. ${ }^{17-22}$ Mandelblatt et al. ${ }^{18}$ found that maximum savings in life could be achieved by screening every 2 years beginning at age 20 years until death with a combination of Pap and human papilloma-virus (HPV) testing. Ending Pap tests at age 75 or 65 years could result in savings while retaining 98\%-87\% of the benefits of lifetime biennial Pap screening. ${ }^{19}$

Although some studies have also examined the extent to which new screening technologies are cost-effective relative to traditional methods, ${ }^{23,24}$ few examined the cost-effectiveness of intervention strategies to increase cervical cancer screening, especially among Hispanic women. A cost-effectiveness study of promotion strategies to increase screening for cervical cancer at the Kaiser Northwest HMO found an initial letter with follow-up phone call was 
more cost-effective than strategies using identical initial and follow-up mechanisms (i.e., initial letter with letter follow-up or initial phone call with phone follow-up). ${ }^{25}$

Hispanic women were not specifically targeted in the Kaiser study. A study of the relative cost-effectiveness of individual versus group lay educator interventions for Hispanic women in Phoenix found little difference in the effect of the interventions and lower cost in the group-level program. ${ }^{26}$ Additional reports about the cost-effectiveness of these interventions will provide researchers, program managers, and policy makers with information to compare, select, and budget for cervical cancer screening interventions.

This paper presents the cost-effectiveness evaluation of the AMIGAS intervention from the payer and payer plus participant perspectives. Although the direct costs may be the main budgetary concern for decision makers, the burden on participants in terms of cost and time is also important to assess as a major component of the full societal cost and a potential factor in the rate of participation in the interventions.

\section{Methods}

The cost-effectiveness analysis was conducted alongside the community-based RCT of the AMIGAS intervention. The primary intent of the study was to measure the effectiveness of key small media components of AMIGAS when used individually or together versus control. The research protocol for this study was approved by the University of Texas Health Science Center-Houston Committee for the Protection of Human Subjects and the Fred Hutchinson Cancer Research Center IRB.

The AMIGAS intervention was designed using Intervention Mapping ${ }^{27}$ and delivered in participants-homes or preferred locations by trained lay health workers (promotoras) in either English or Spanish from 2008 through 2011. Promotoras are from the community being served and have some training (usually a series of courses) but are not professional health workers.

They typically have similar demographic and cultural characteristics as the people they serve and act as trusted models and health educators. In Texas, many lay health workers are -certified-by the state health department by taking approved classes and continuing education. They are paid substantially less than health educators with a BS or MS degree or nurses.

Promotoras received detailed instructions to guide their use of educational materials in describing cervical cancer, related risk factors, benefits of screening, and the screening process. The AMIGAS intervention includes a video that portrays women from the community addressing common barriers and beliefs about cervical cancer screening, a flipchart with additional information for reinforcing the video, and handouts that can be used at the discretion of the promotora to further reinforce the messages.

The trial was conducted in El Paso and Houston TX and the Yakima Valley WA. Women of Mexican descent aged $\geq 21$ years with no history of cancer, no hysterectomy, and no cervical cancer screening within the past 3 years were eligible. Further details about the recruitment 
process and demographic characteristics of the women in each intervention site are presented elsewhere. ${ }^{16}$

Following a baseline interview, 613 eligible women were randomly assigned to one of three intervention arms or a control arm (Figure 1). Study arms differed by the types of materials the promotoras used to deliver the program, flipchart, a video, or both the flipchart and video. These versions of AMIGAS were compared to a control group. Women in the control group may have received cervical cancer screening education in clinics but did not receive promotora-led education.

The follow-up interview occurred at least 6 months after the intervention to assess whether participants received a Pap test since the initial contact. Regardless of how the participants answered (yes or no), receipt or non-receipt of Pap test was confirmed with the identified clinic. 15,16

\section{Cost-Effectiveness Analysis}

The economic analysis was conducted during 2012-2013 to provide information on the costeffectiveness of the AMIGAS interventions. The economic evaluation includes data and an analytic framework for decision making from both the participant and provider perspectives.

Participants are persons in the trial who are the targets of the screening promotion interventions. Providers are clinics or health agencies that are responsible for paying for the screening-promotion interventions. The analysis assumes that providers are responsible for all resource costs of the intervention but not participant time cost. Intent to treat-based point estimates of cost and screening outcome were applied for the comparison of groups. This conservative strategy analyzes results for all randomized participants.

If outcome data were missing, screening was assumed to not have occurred. Considerable evidence supports the effectiveness and cost-effectiveness of cervical cancer screening. 5 Therefore, the task was to estimate the cost-effectiveness of this evidence-based intervention designed to increase cervical cancer screening, not to provide evidence of the costeffectiveness of screening tests.

The primary measure of cost-effectiveness was the incremental cost effectiveness ratio (ICER). ICERs were obtained by dividing the additional per target individual cost by the additional percentage of target individuals who were screened by the end of the follow-up period, comparing the control group to the video group, the flipchart group, and the video plus flipchart group, moving from the least costly to the most costly intervention. For example, equation (1) represents the ICER calculation comparing the video arm to the control arm:

$$
\mathrm{ICER}=\left(\mathrm{Cost}_{\text {Video }}-\text { Cost }_{\text {Control }}\right) \div\left(\mathrm{OScrned}_{\text {Video }}-\% \text { Scrned }_{\text {Control }}\right)
$$

where the overbar represents mean cost in the study arm and \% represents the percentage of persons screened in the study arm. 


\section{Effectiveness}

The primary outcome (one-time Pap test at the 6-month follow-up) was measured among participants who previously had not adhered to recommended screening guidelines. Receipt of Pap test was evaluated under four scenarios: (1) those receiving the full AMIGAS intervention, which includes both the flipchart and video; (2) those receiving the AMIGAS with the flipchart only (no video); (3) those receiving the AMIGAS with the video only (no flipchart); and (4) those in the control group (Figure 1).

\section{Costs}

Micro-costing (tracking resource use and weighting by their prices) was selected over gross costing (tracking final services and weighting by their prices). Although micro-costing was labor intensive, it was the most precise form of cost determination and recommended by the U.S. Panel on Cost-Effectiveness Analysis. ${ }^{28}$

Costs included were those for planning, training staff, recruiting eligible women to receive the intervention, and delivering the intervention. Resource unit prices were obtained from project administrative records, and participant time was valued by the median full-time weekly earnings for Hispanic women. ${ }^{29}$ Promo-tora visit time and travel distance were tracked via encounter and travel reimbursement logs. Participant time was tracked via promotora encounter forms. Travel cost was estimated with the Internal Revenue Service estimate of cost per mile traveled. ${ }^{30}$

Overhead was estimated at $35 \%$ of direct cost, following previous standards used in community health prevention studies. ${ }^{31}$ The overhead estimate covers office space, utilities, housekeeping, basic office equipment, and administration by a manager who is responsible for the overall community health program. Project records were used to itemize, quantify, and value multimedia equipment and supplies. Cost estimates were in 2008 U.S. dollars.

\section{Sensitivity Analysis}

Estimates and methods uncertainty were evaluated with sensitivity analysis to determine effects of alternative parameter estimates and methods on the ICERs. Key parameters for sensitivity analysis included the overhead cost rate and size of the target population with a range of 300 to 5,000, assuming fixed costs for training staff, planning meetings, coordinator, and materials over that range. Costs and effect were not discounted owing to the short duration of the program.

Because the ICER is a ratio statistic with the potential for a zero denominator, the variance formula necessary to compute CIs becomes more difficult. Alternatively, the nonparametric bootstrap resampling method (1,000 re-samples) was used to obtain $95 \%$ confidence limits for the ICERs. ${ }^{32}$ SAS/STAT, version 9.2 (SAS Institute Inc., Cary NC), was used to conduct the bootstrap analysis.

\section{Results}

The intention-to-treat results for 613 participants in the AMIGAS RCT were utilized for the cost-effectiveness analysis. Participants were aged 21-66 years with an median age of 38 
years, $87.6 \%$ were born in Mexico, lived 16 years in the U.S. on average, $68.6 \%$ were married, $93.0 \%$ were ever pregnant, $89.0 \%$ ever had a pap test, and $18.1 \%$ reported some healthcare coverage. ${ }^{16}$ The three intervention arms yielded significantly higher screening rates compared to the $24.8 \%$ screening rate achieved in the control arm.

The screening rate for participants in the full AMIGAS arm (52.3\%) was 6.8\% higher than for the flipchart-only arm and $11.0 \%$ higher than for the video-only arm, although the differences between the interventions did not reach statistical significance at the 0.05 level. ${ }^{16}$ Results from this study demonstrated that the per person cost of full, flipchart-only, and video-only interventions were $\$ 223, \$ 219$, and $\$ 216$, respectively, from the payer plus participant perspective; and $\$ 165, \$ 165$, and $\$ 162$ respectively, from the payer perspective (Table 1).

From the payer plus participant perspective, this distribution of cost was similar across the three arms: cost for the payer plus participant perspective included participant time (25\%); recruitment (23\%); conducting the intervention (21\%); local travel (16\%); and staff training $(8 \%)$. From the payer perspective, about $17 \%$ of the cost was for recruitment, $16 \%$ for conducting the intervention, $21 \%$ for local travel, and $10 \%$ for training.

Table 2 shows the cost per additional person screened or the ICER from the payer plus participant perspective was $\$ 1,308$ (95\% CI=\$869, $\$ 2,396)$ comparing no intervention to the video intervention; $\$ 76$ (95\% CI=-\$7, \$7) comparing the video to flip-chart arm; and \$57 ( $95 \% \mathrm{CI}=-\$ 13, \$ 6)$ comparing the flipchart to the full intervention arm. The declining ICERs as interventions become more costlyimply that screening of additional women can be achieved with minimal additional cost compared to the next less costly intervention.

For example, given that it costs only $\$ 3.90$ more per person to deliver the full intervention arm compared to the flipchart-only arm and $6.8 \%$ more participants get screened, the cost per additional woman screened is only $\$ 57$. Each of the 1,000 bootstrap samples yielded a cost-effectiveness ratio with effect and cost being higher in the intervention groups compared to the control group. In no sample was the cost of the video intervention higher and effect lower than the control group.

The ICER computed from the payer perspective only, excluding participant cost, was $\$ 980$ $(95 \% \mathrm{CI}=\$ 650, \$ 1,974)$ comparing controls to the video-only intervention; $\$ 71$ (95\% CI=$\$ 4.10, \$ 6.60)$ comparing the video-only to flipchart-only arm; and $\$ 4(95 \% \mathrm{CI}=-\$ 1.80$, $\$ 1.60$ ) comparing the flipchart-only to the full intervention arm (Table 2).

The ICER declined at a decreasing rate when expanding the intervention to a larger population with the same level of fixed costs (planning, staff training, project coordinator, and others) incurred in the trial when the target population was hypothetically increased from 600 to 1,250; the estimated payer cost per additional woman screened using the video declined from $\$ 908$ to $\$ 811$ and to $\$ 685$ when the target population was 5,000 women (data not shown).

Alternative assumptions for the overhead rate had minimal effect on the ICERs. For example, increasing the overhead rate from $30 \%$ to $40 \%$ increased the incremental cost per 
additional woman receiving a Pap test by approximately $6 \%$ when comparing the video-only arm to the controls in both payer perspective and the societal perspective analyses.

\section{Discussion}

The AMIGAS intervention produced a statistically significant increase in cervical cancer screening rates among low-income women of Mexican descent in diverse urban and rural settings who had never or rarely been screened. The cost per additional women screened comparing the video-only version of the program to controls was approximately $\$ 980$. The cost increased to $\$ 1,309$ when participant time was included in the cost estimates.

If these costs are considered feasible and of value, program decision makers may consider employing the full AMIGAS intervention. With a minimal additional cost of $\$ 3.90$ per participant compared to the flipchart-only arm, the full AMIGAS intervention yields $6.8 \%$ additional women screened.

The study results clearly demonstrate, however, that the video- and the flipchart-only conditions are also successful in increasing cervical cancer screening among women of Mexican descent. ${ }^{16}$ Likewise, our analysis demonstrated that the small media components of the AMIGAS intervention are cost-effective methods of increasing cervical cancer screening in this population.

These cost findings should be considered an upper bound in replicating AMIGAS in nonresearch settings. The program was delivered in the participant-s home, resulting in substantial travel cost. Depending upon the context, home visits may present safety challenges. AMIGAS training includes guidance about promotoras-safety, including delivery in pairs, which increases cost. As providers gain additional experience and become more efficient in delivering AMIGAS, the program effect may be enhanced and costs may be lowered.

Given that the cost of recruitment for prevention programs ranges from $20 \%$ to $40 \%$ of the cost of implementation, ${ }^{33}$ decision makers may consider leveraging AMIGAS to incorporate additional cancer prevention services. This strategy may reduce the cost per prevention behavior outcome by a factor dependent on the number of targeted behaviors and additional costs incurred because of greater complexity of the intervention.

Chirikos et al. ${ }^{34}$ described an economic analysis of a multi-prevention service intervention whereby implementing a central clinic reminder system targeting compliance with screening for breast, cervical, and colorectal cancer reduced the marginal cost of achieving compliance with an additional test. They apportioned the cost of the intervention over the sample, depending on the distribution of tests (one, two, or three) indicated for the patients in the sample.

Programs with a similar level of fixed inputs that serve more people may reduce average cost when replicating AMIGAS by spreading the fixed costs incurred for initial planning, staff training, and project coordination. As in most studies, the trial was sized to measure the effect and not for maximum efficiency. The planning and training costs can be spread over 
more participants, although the maximum number that could be recruited and served without expanding the fixed inputs is unknown.

The reader is therefore cautioned that the simulation is intended to provide an indication of how the ICER may decline with increased target population. Alternatively, attention should be given to the level of difficulty in reaching additional women, which may require additional coordinators and associated training costs, thereby reducing the efficiency gains of serving a larger group.

The most relevant comparison of the findings to the cost-effectiveness literature is with the Phoenix study of cancer screening interventions for underserved Latinas. ${ }^{26}$ This study found that interventions to increase screening for breast, cervical, and colorectal cancers averaged $\$ 103$ in 2006 U.S. dollars when delivered to groups and $\$ 392$ when delivered to individuals, both from the perspective of the sponsoring organization.

Larkey and colleagues ${ }^{26}$ estimated an average cost of $\$ 516$ per screening for the group strategy and $\$ 1,716$ per screening for the individual strategy, under the conservative assumption of intention-to-treat. The efficiency of the group strategy was further supported by considering the imputed ICER. The incremental cost per additional screening was $\$ 4,736$ when moving from the group to the individual strategy. ${ }^{26}$ These findings compare favorably to a cost per additional screening of $\$ 980$ in this study, when comparing the control group to the video-only strategy, clearly indicating a more efficient one-on-one intervention.

Although AMIGAS was designed for one-on-one and group formats, further research is needed to determine the effectiveness of the group format. Nevertheless, the use of evidence-based interventions such as AMIGAS with groups may improve the odds of having an effective intervention when applied in settings that were not included in the original study.

Two additional studies provide comparison for estimates of cost effectiveness. A study of phone- and letter-based interventions to increase cervical cancer screening at the Kaiser Permanente Northwest health plan found a cost per additional screening that ranged from $\$ 185$ (letter with phone follow-up) to $\$ 1,117$ (letter with letter follow-up) in 1996 U.S. dollars. ${ }^{25}$ This equals about $\$ 254$ and $\$ 1,533$ in 2008 prices. ${ }^{35}$ Each intervention was compared to a no intervention, zero cost control group.

A direct mail versus outreach intervention study to increase cervical cancer screening among Chinese American women in Seattle and Vancouver found a cost per additional screening that ranged from $\$ 415$ (outreach) to $\$ 676$ (direct mail) in 1999 U.S. dollars. This equals about $\$ 536$ and $\$ 874$ in 2008 prices. Each comparison was based on intent-to-treat and compared to a no intervention, zero cost control group. ${ }^{36}$

Limitations to the cost-effectiveness analysis include self-report of personnel time and screening outcome, and the application of an assumed overhead cost rate. Consistency of personnel time estimates across study sites (data not shown) suggest that relative costs were not distorted by measurement error. Medical chart reviews showed that the validity of selfreported screening outcome in this study was comparable or better than that found in other 
studies where self-report of Pap test screening was validated by clinical record review. ${ }^{16}$ The overhead is based on previously reported standard rates and is applied equally to each study arm, minimizing the effect of measurement error on the relative cost of the interventions.

Strengths of the study include the concurrent conduct of the economic evaluation with a community-based RCT, inclusion of the payer and payer plus participant perspectives, and direct micro-costing of the planning and operating costs of the intervention. ${ }^{37}$ Inclusion of three geographically dispersed sites further enhances the generalizability of the findings for the intended population of low-income women of Mexican descent.

In summary, a screening test for cervical cancer saves lives and is cost-effective, ${ }^{18}$ but lowincome women of Mexican descent are disproportionately underscreened. ${ }^{1,7,8}$ The effectiveness and cost-effectiveness of the AMIGAS intervention suggest value in developing evidence-based interventions with and for populations that are traditionally medically underserved. This study adds important economic evaluation information to the limited body of evidence on evidence-based cervical cancer screening, which may assist health policy makers and program managers select among and budget for programs to enhance screening for cervical cancer and achieve lower mortality rates among low-income women of Mexican descent.

\section{Acknowledgments}

Research for this publication was supported by the CDC cooperative agreement U48-DP000057 to the University of Texas, School of Public Health at El Paso. The findings and conclusions in this report are those of the authors and do not necessarily represent the official position of the CDC.

\section{References}

1. Coughlin SS, Uhler RJ, Richards T, Wilson KM. Breast and cervical cancer screening practices among Hispanic and non-Hispanic women residing near the U.S-Mexico border, 1999-2000. Fam Community Health. 2003; 26(2):130-9. [PubMed: 12802118]

2. Simard EP, Fedewa S, Ma J, Siegel R, Jemal A. Widening socioeconomic disparities in cervical cancer mortality among women in 26 states, 1993-2007. Cancer. 2012; 118(20):5110-6. [PubMed: 22707306]

3. Freeman, HP.; Wingrove, BK. Excess cervical cancer mortality: a marker for low access to health care in poor communities. Rockville MD: National Cancer Institute, Center to Reduce Cancer Health Disparities; 2005. NIH Pub. No. 05-5282crchd.cancer.gov/attachments/excesscervcanmort.pdf

4. Screening for cervical cancer: clinical summary of U.S. Preventive Services Task Force recommendation. Rockville MD: U.S. Preventive Services Task Force; Agency for Healthcare Research and Quality; Moyer VA: for the U S. Preventive Services Task Force; 2012. Publication No. 11-05156-EF-3uspreventiveservicestaskforce.org/uspstf11/cervcancer/cervcancersum.htm

5. Screening for cervical cancer: U.S Preventive Services Task Force recommendation statement. Ann Intern Med. 2012; 156(12):880-91. [PubMed: 22711081]

6. U.S. Cancer Statistics Working Group. U.S. cancer statistics: 1999-2009 incidence and mortality web-based report. Atlanta GA: USDHHS, CDC, National Cancer Institute; 2013. cdc.gov/uscs

7. Bustamante AV, Chen J, Rodriguez HP, Rizzo JA, Ortega AN. Use of preventive care services among Latino subgroups. Am J Prev Med. 2010; 38(6):610-9. [PubMed: 20494237] 
8. U.S.-Mexico Border Health Commission. Healthy Border 2010: an agenda for improving health on the U.S.-Mexico Border. El Paso TX: U.S.-Mexico Border Health Commission; borderhealth.org/ reports.php?curr=about_us

9. USDHHS. Healthy People 2020. healthypeople.gov/2020/topicsobjectives2020/objectiveslist.aspx? topicId $=5$

10. U.S. Centers for Medicare \& Medicaid Services. What are my preventive care benefits?. https:// www.healthcare.gov/what-are-my-preventive-care-benefits/

11. U.S. Preventive Services Task Force. Screening for Cervical Cancer: Clinical Summary of U.S. Preventive Services Task Force Recommendation. Mar. 2012 AHRQ Publication No. 11-05156EF-3http://www.uspreventiveservicestaskforce.org/uspstf11/cervcancer/cervcancersum.htm

12. Baron RC, Rimer BK, Breslow RA, et al. Interventions to increase community demand for breast, cervical, and colorectal cancer screening: a systematic review. Am J Prev Med. 2008; 35(1S):S34S55. [PubMed: 18541187]

13. Sabatino SA, Lawrence B, Elder R, et al. Effectiveness of interventions to increase screening for breast, cervical, and colorectal cancers: nine updated systematic reviews for The Guide to Community Preventive Services. Am J Prev Med. 2012; 43(1):765-86.

14. Community Preventive Services Task Force. Updated recommendations for client- and provideroriented interventions to increase breast, cervical, and colorectal cancer screening. Am J Prev Med. 2012; 43(1):760-4.

15. Byrd TL, Wilson KM, Smith JL, et al. Using intervention mapping as a participatory strategy: development of a cervical cancer screening intervention for Hispanic women. Health Educ Behav. 2012; 39(5):603-11. [PubMed: 22388451]

16. Byrd TL, Wilson KM, Smith JL, et al. AMIGAS: a multicity, multi-component cervical cancer prevention trial among Mexican American women. Cancer. 2013; 119(7):1365-72. [PubMed: 23280399]

17. Gyrd D, Holund B, Anderson P. A cost-effectiveness analysis of cervical cancer screening: health policy implications. Health Policy. 1995; 34(1):35-51. [PubMed: 10151964]

18. Mandelblatt JS, Lawrence WF, Womack SM, et al. Benefits and costs of using HPV testing to screen for cervical cancer. JAMA. 2002; 287(18):2372-81. [PubMed: 11988058]

19. Montz FJ, Farber FL, Bristow RE, Cornelison T. Impact of increasing Papanicolaou test sensitivity and compliance: a modeled cost and outcomes analysis. Obstet Gynecol. 2001; 97(5 pt 1):781-8. [PubMed: 11339934]

20. van Ballegooijen M, van den Akker-van Marle ME, Warmerdam PG, et al. Present evidence on the value of HPV testing for cervical cancer screening: a model-based exploration of the (cost-) effectiveness. Br J Cancer. 1997; 76(5):651-7. [PubMed: 9303366]

21. van Ballegooijen M, van den Akker-van Marle ME, Patnick J, et al. Overview of important cervical cancer screening process values in European (EU) countries, and tentative predictions of the corresponding effectiveness and cost-effectiveness. Eur J Cancer. 2000; 36(17):2177-88. [PubMed: 11072201]

22. van den Akker-van Marle ME, van Ballegooijen M, van Oortmarssen G, et al. Cost-effectiveness of cervical cancer screening. J Natl Cancer Inst. 2002; 94(3):193-204. [PubMed: 11830609]

23. Holmes J, Hemmett L, Garfield S. The cost-effectiveness of human papillomavirus screening for cervical cancer. Eur J Health Econ. 2005; 50(1):30-7. [PubMed: 15682286]

24. Kim JJ, Wright TC, Goldie SJ. Cost-effectiveness of human papillomavirus DNA testing in the United Kingdom, The Netherlands, France, and Italy. J Natl Cancer Inst. 2005; 97(12):888-95. [PubMed: 15956650]

25. Vogt TM, Glass A, Glasgow RE, La Chance PA, Lichtenstein E. The safety net: a cost-effective approach to improving breast and cervical cancer screening. J Womens Health (Larchmt). 2003; 12(8):789-98. [PubMed: 14588129]

26. Larkey LK, Herman PM, Roe DJ, et al. A cancer screening intervention for underserved Latina women by lay educators. J Womens Health (Larchmt). 2012; 21(5):557-66. [PubMed: 22416791]

27. Bartholomew, LK.; Parcel, G.; Kok, G.; Gottlieb, N. Planning health promotion programs: an intervention mapping approach. San Francisco CA: Jossey-Bass; 2006. 
28. Gold, MR.; Siegel, JE.; Russell, LB.; Weinstein, MC., editors. Cost effectiveness in health and medicine. New York: Oxford University Press; 1996. Estimating costs in cost-effectiveness analysis.

29. U.S. Department of Labor. Usual weekly earnings of wages and salaried workers: second quarter 2008. Washington DC: Bureau of Labor Statistics; 2008.

30. U.S. Internal Revenue Service. IRS 2008 Standard Mileage Rates. irs.gov/uac/IRSAnnounces-2008-Standard-Mileage-Rates;-Rate-for-Business-Miles-Set-at-50.5-Cents-per-Mile

31. Andersen MR, Hager M, Su C, Urban N. Analysis of the cost-effectiveness of mammography promotion by volunteers in rural communities. Health Educ Behav. 2002; 29(6):755-70. [PubMed: 12456132]

32. Briggs AH, O-Brien BJ, Blackhouse G. Thinking outside the box: recent advances in the analysis and presentation of uncertainty in cost-effectiveness studies. Annu Rev Public Health. 2002; 23:377-401. [PubMed: 11910068]

33. Ritzwoller DP, Sukhanova A, Gaglio B, Glasgow RE. Costing behavioral interventions: a practical guide to enhance translation. Ann Behav Med. 2009; 37(2):218-27. [PubMed: 19291342]

34. Chirikos TN, Christman LK, Hunter S, Roetzheim RG. Cost-effectiveness of an intervention to increase cancer screening in primary care settings. Prev Med. 2004; 39(2):230-8. [PubMed: 15226030]

35. U.S. Department of Labor, Bureau of Labor Statistics. CPI Inflation Calculator. bls.gov/data/ inflation_calculator.htm

36. Thompson B, Thompson LA, Chan NL, Hislop TG, Taylor VM. Cost effectiveness of cervical cancer screening among Chinese women in North America. Asian Pac J Cancer Prev. 2007; 8(2): 287-93. [PubMed: 17696748]

37. Fryback DG, Craig BM. Measuring economic outcomes of cancer. J Natl Cancer Inst Monogr. 2004; 33:134-41. [PubMed: 15504924] 




Figure 1.

AMIGAS study design

Note: Permission to reproduce this figure has been granted by John Wiley and Sons, March 21, 2013, under license number 3113681391547. AMIGAS, Ayudando a las Mujeres con Información, Guia, y Amor para su Salud 


\section{Table 1}

Average cost by study arm, $\$$

\begin{tabular}{|c|c|c|c|}
\hline Cost category & Full (video + flipchart; $n=134$ ) & Video $(n=144)$ & Flipchart $(n=146)$ \\
\hline \multicolumn{4}{|l|}{ Payer and participant } \\
\hline Staff time for recruitment & 28.20 & 28.20 & 28.20 \\
\hline Participant time for recruitment & 21.30 & 21.30 & 21.30 \\
\hline Staff time for intervention & 28.30 & 24.80 & 24.90 \\
\hline Participant time for intervention & 21.60 & 18.80 & 19.00 \\
\hline Staff time for travel & 17.70 & 18.90 & 20.60 \\
\hline Mileage for travel & 15.50 & 15.80 & 16.80 \\
\hline Training for staff & 16.90 & 16.90 & 16.90 \\
\hline Meetings for staff & 7.90 & 7.90 & 7.90 \\
\hline Coordinator time & 8.90 & 8.90 & 8.90 \\
\hline Material for interventions & 7.50 & 7.50 & 7.50 \\
\hline Average direct cost & 173.80 & 168.90 & 172.0 \\
\hline Overhead $^{a}$ & 49.20 & 47.00 & 47.10 \\
\hline Total average cost & 223.00 & 215.90 & 219.10 \\
\hline \multicolumn{4}{|l|}{ Payer } \\
\hline Average direct cost & 130.90 & 128.80 & 131.70 \\
\hline Overhead $^{b}$ & 34.20 & 32.90 & 33.00 \\
\hline Total average cost & 165.00 & 161.70 & 164.70 \\
\hline
\end{tabular}

$a_{35 \% \text { of direct cost, excluding travel }}$

$b_{35 \% \text { of direct cost, excluding participant cost and travel }}$ 


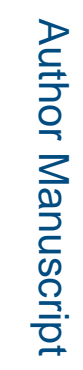



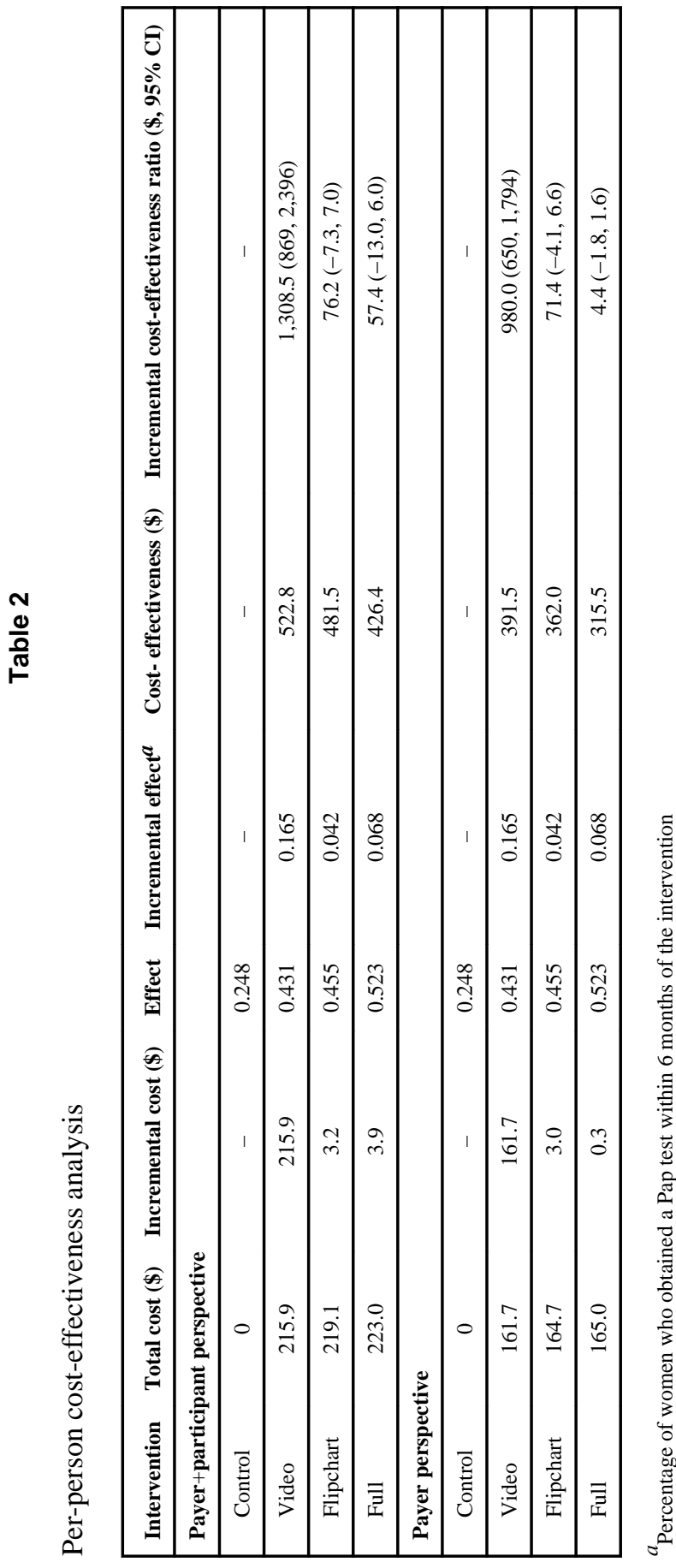

要

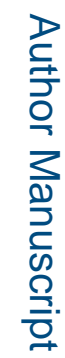

Am J Prev Med. Author manuscript; available in PMC 2015 October 13. 